\title{
Meta-Analysis on Efficacy and Safety of Modified Guizhi Shaoyao Zhimu Tang with the Western Medicines in Treating Rheumatoid Arthritis
}

\author{
Wenyi Lu¹, Zushan Zhou², Syed Manzar Abbas Shah Naqvi', Xiaolin Li1 \\ ${ }^{1}$ Department of Medical College, Yangtze University, Jingzhou, China \\ ${ }^{2}$ The Affiliated Hospital of Yangtze University, Honghu Hospital of Traditional Chinese Medicine, Jingzhou, China \\ Email: 925812706@qq.com
}

How to cite this paper: Lu, W.Y., Zhou, Z.S., Naqvi, S.M.A.S. and Li, X.L. (2018) Meta-Analysis on Efficacy and Safety of Modified Guizhi Shaoyao Zhimu Tang with the Western Medicines in Treating Rheumatoid Arthritis. Yangtze Medicine, 2, 161-170. https://doi.org/10.4236/ym.2018.23017

Received: June 10, 2018

Accepted: August 27, 2018

Published: August 30, 2018

Copyright (c) 2018 by authors and Scientific Research Publishing Inc. This work is licensed under the Creative Commons Attribution International License (CC BY 4.0).

http://creativecommons.org/licenses/by/4.0/

\begin{abstract}
Objective: To assess the efficacy and safety of modified Guizhi Shaoyao Zhimu Tang (GSZT) with the Western medicines in treating rheumatoid arthritis. Methods: All the relevant studies were collected by searching the Pubmed, Web of science, Embase, Cochrane Library, SpringerLink, China National Knowledge Infrastructure (CNKI), VIP Journals database, and Wanfang database from their inception to October 2017. After the assessment, the eligible ones were subject to Jadad score. The meta-analysis was conducted by RevMan 5.3 software. Results: Totally 10 randomized controlled trials (RCTs) involving 962 patients were enrolled. The clinical efficacy of analysis showed that modified GSZT with the Western medicines can significantly improve the clinical total effective rate $[\mathrm{OR}=4.64,95 \% \mathrm{CI}(3.03$, 7.12), $\mathrm{P}<0.00001]$, the duration of morning stiff $[\mathrm{MD}=-14.58,95 \% \mathrm{CI}$ $(-16.49,-12.68), \mathrm{P}<0.00001]$, erythrocyte sedimentation rate (ESR) $[\mathrm{MD}=$ $-12.6295 \% \mathrm{CI}(-18.69,-6.54), \mathrm{P}<0.001]$, rheumatoid factor $(\mathrm{RF})[\mathrm{MD}=$ $-49.88,95 \% \mathrm{CI}(-77.6,-22.09), \mathrm{P}<0.001]$, C-reactive protein $(\mathrm{CRP})[\mathrm{MD}=$ $-5.34,95 \% \mathrm{CI}(-5.86,-4.82), \mathrm{P}<0.001]$. The adverse events had been seen in both of the two groups, however, the clinical symptoms of the group on modified GSZT with the Western medicines were obviously reduced than the other one $[\mathrm{OR}=0.25,95 \% \mathrm{CI}(0.10,0.61), \mathrm{Z}=3.06, \mathrm{P}<0.01]$. This system review shows that the modified GSZT with the Western medicines improved the clinical efficacy, symptoms and the blood biochemical indicators were improved significantly than that of Western medicines alone on rheumatoid arthritis. At the same time, it can reduce adverse effects and enhance safety.
\end{abstract}




\section{Keywords}

Rheumatoid Arthritis, Guizhi Shaoyao Zhimu Tang, RA, Meta

\section{Introduction}

Rheumatoid arthritis (RA) is a chronic autoimmune disease that is characterized by joint erosion, stiffness, deformity, loss of function, and with high morbidity [1] [2]. However, the cause of RA is unknown. RA belongs to the category of "bi syndrome" in Traditional Chinese Medicine (TCM). It is caused by invading cold and dampness inside body and blood qi stasis in the theory of TCM. At present, the effective treatments of routine western drugs include glucocorticoid, non-steroidal anti-inflammatory drugs (NSAIDs), disease modifying anti-rheumatic drugs (DMARDs) and biologic agents. While the long term use of these drugs can produce the multiple side effects, and cause much socioeconomic costs and safety, and even some patients cannot tolerate the situation [3]. But the TCM has special effects in the treatment of RA [4]. Recently, the TCM is attracting the attention of the public, because of the potential and positive effects in treatment of autoimmune disease. The TCM mainly contains Chinese herbs. In the ancient period of China, there was a formula that was most commonly used in the management of RA. Guizhi Shaoyao Zhimu Tang (GSZT) is the classical Chinese medicine which is extensively used in clinical application. In this article, the potentially therapeutic effect and safety of the GSZT combined with the Western medicine for the treatment of RA were systematically evaluated.

\section{Materials and Methods}

\subsection{Literature Retrieval Strategy}

The search strategy was developed by Cochrane collaboration and the relevant studies were collected by searching the Pubmed, Web of science, Embase, Cochrane Library, SpringerLink, CNKI, VIP Journals database, and Wanfang database from their inception to October 2017. A multiple number of medical terms such as "rheumatoid arthritis", "RA" and "Guizhi Shaoyao Zhimu Tang" were utilized for medical subject heading $(\mathrm{MeSH})$ to find out the articles, which have been published on the base of combine conventional western medicines for the treatment of RA. The retrieval is conducted in the form of free combination with a keyword, manual retrieval had been used to trace the references in the literature. No language or time restrictions were applied in the search.

\subsection{Document Inclusion and Exclusion Criteria}

Trials were considered to be eligible for research propose if they fully meet the following criteria: 1) all patients fulfilled the1987 revised American College of 
Rheumatology criteria for the disease [5], 2) the experimental group was treated with the combined conventional Western medicine and the GSZT, and the control group was just treated with conventional Western medicine, 3) the research type satisfied the RCT data, 4) the outcomes included at least one of the following therapeutic effects: duration of morning stiffness (DMS), erythrocyte sedimentation rate (ESR), rheumatoid factor (RF), C-reactive protein (CRP), there were no restrictions regarding gender, age, severity or duration of RA. The safety evaluation index is the adverse reactions.

\subsection{Data Extraction}

The two researchers independently screened and extracted the retrieved documents, and the differences were determined and resolved by the third person, including study design, randomization and diagnostic criteria.

\subsection{Quality Evaluation}

According to the quality criteria of Cochrane Review's Handbook, the literature quality is evaluated and Jadad scale method was independently used for the evaluation of methodological quality of articles [6]. The specific contents include: 1) random grouping sequence method, 2) blind method, include double-blind method and single blind method, 3) allocation concealment, 4) reports about the withdrawal.

\subsection{Statistical Analysis}

Data analysis was performed by using the RevMan 5.3 software provided by the Cochrane collaboration. The statistical data were analyzed by odds ratio (OR) and $95 \%$ confidence intervals (CI) and the metering data was expressed by mean difference (MD) and 95\%CI. A fixed-effect model was employed if there was no statistical heterogeneity among studies, otherwise, the random effect model was used [7]. Heterogeneity is assessed by Chi-square test, and the degree of inconsistency Higgins $\mathrm{I}^{2}$ is measured between studies whether the percentage of total variation across studies is due to heterogeneity rather than chance [8]. If the source of heterogeneity is unable to be determined, the descriptive analysis will be adopted. The distribution of the research data, in the study, was analyzed by funnel plot. The reliability analysis of publication bias was carried out.

\section{Results}

\subsection{Study Selection and Characteristics}

According to the above mentioned research strategy we got information from 1313 articles which are potentially relevant. 1078 studies were screened after removal of duplicates. There were ten relevant studies that finally met the inclusion criteria for the meta-analysis. The general situation of the study selection was shown in Figure 1. 


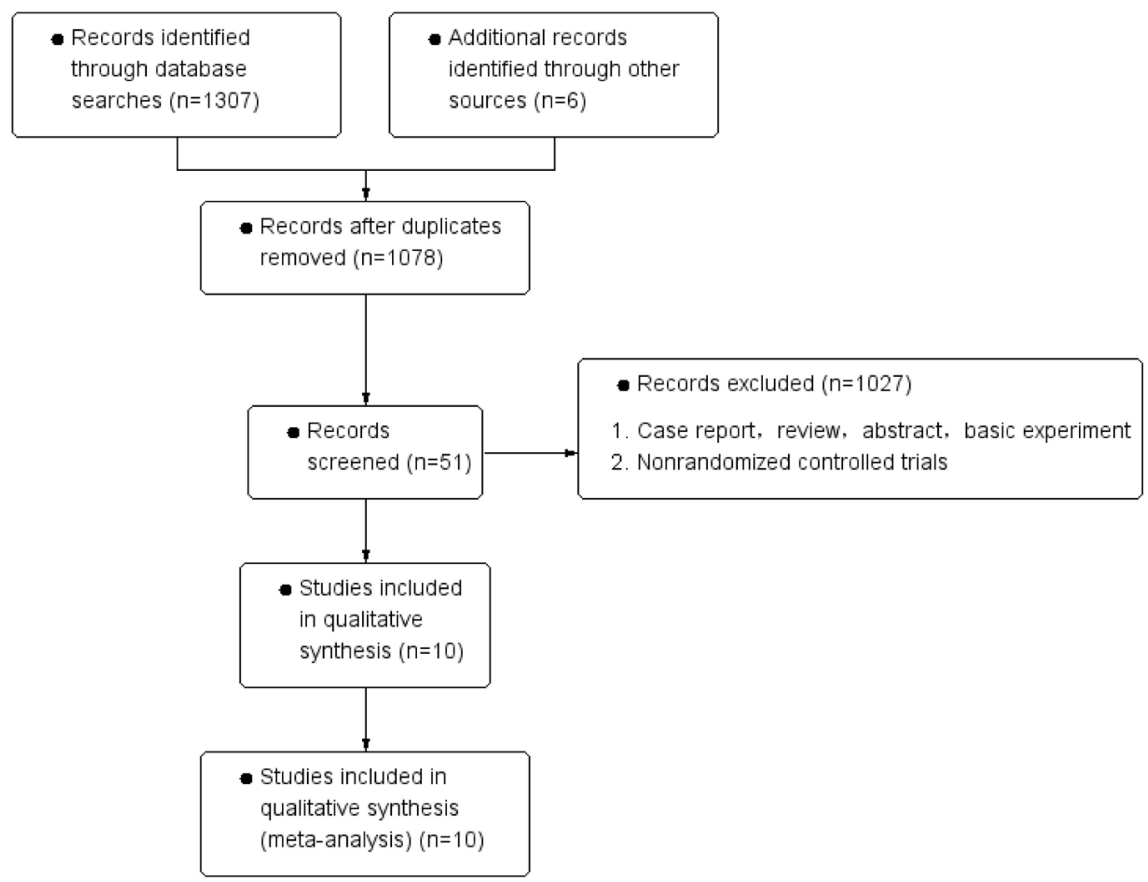

Figure 1. Flow diagram of study selection process.

\subsection{General Situation and Quality Evaluation of the Included Literature}

A total of 10 RCTs were included [9]-[18], and the conventional Western medicine involves three major categories in these studies. They are non-steroidal anti-inflammatory drugs (NSAIDs), disease modifying anti-rheumatic drugs (DMARDs) and glucocorticoids (GCs). NSAIDs included Meloxicam (MLX), voltaren, nimesulide (Nime), celecoxib (CXB), loxoprofen. DMARDs included methotrexate (MTX), sulfasalazine (SSZ) and Leflunomide (LEF). The experimental group was based on the control group with GSZT. The general description about literature and the results of methodological quality evaluation are shown in Table 1.

\subsection{Meta Analysis Results}

\subsubsection{Comparison of Disease Efficacy for RA}

In the articles that met the criteria. 9 trials were compared for the efficacy of RA patients and the specific values were given [9]-[17]. According to the data which we had screened in combination, we can see that 9 trials included in the study were homogeneity (no heterogeneity) and showed insignificant difference $\left(\mathrm{X}^{2}=\right.$ $\left.1.57, \mathrm{P}=0.99, \mathrm{I}^{2}=0 \%\right)$. The fixed effect model was used and showed $[\mathrm{OR}=4.64$, 95\%CI $(3.03,7.12), \mathrm{Z}=7.04, \mathrm{P}<0.00001]$. Therefore, it is considered that there is a statistically significant difference in the efficacy of the combination therapy between the combination therapy trial group and the Western medicine treatment control group alone. In addition, the lower and lower limit of 95\%CI of OR is more than 1. It can be seen that the clinical efficacy of the experimental group is better than the Western medicine alone control group in RA. See Figure 2. 
Table 1. Characteristics of the ten trials included in the meta-analysis.

\begin{tabular}{|c|c|c|c|c|c|c|c|c|c|}
\hline \multirow{2}{*}{ Author } & \multirow{2}{*}{$\begin{array}{l}\text { random } \\
\text { allocation }\end{array}$} & \multirow{2}{*}{$\begin{array}{l}\text { allocation } \\
\text { concealment }\end{array}$} & \multirow{2}{*}{$\begin{array}{l}\text { blind } \\
\text { method }\end{array}$} & \multirow{2}{*}{ withdraw } & \multirow{2}{*}{$\begin{array}{l}\text { Jadad } \\
\text { score }\end{array}$} & \multicolumn{2}{|c|}{ Interventions } & \multirow{2}{*}{ Duration } & \multirow{2}{*}{ Outcomes } \\
\hline & & & & & & experimental & control & & \\
\hline Yu 2010 [9] & yes & unclear & unclear & unclear & 3 & GSZT & $\begin{array}{l}\text { MTX } 7.5 \mathrm{mg} \mathrm{qw} \\
\text { SSZ } 1 \mathrm{~g} \text { bid }\end{array}$ & 12 weeks & $\begin{array}{c}\text { TE, DMS, } \\
\text { ESR, CRP, AE }\end{array}$ \\
\hline Liu 2008 [10] & unclear & unclear & unclear & unclear & 2 & GSZT & $\begin{array}{l}\text { MTX } 7.5 \mathrm{mg} \mathrm{qw} \\
\text { MLX7.5 mg bid }\end{array}$ & 4 weeks & TE \\
\hline Liu 2008 [11] & unclear & unclear & unclear & unclear & 2 & GSZT & LEF $20 \mathrm{mg}$ qd & 4 weeks & TE \\
\hline Lv 2014 [12] & yes & unclear & unclear & unclear & 3 & GSZT & $\begin{array}{l}\text { MTX } 7.5 \mathrm{mg} \mathrm{qw} \text {, } \\
\text { voltaren } 75 \mathrm{mg} \text { qd }\end{array}$ & 12 weeks & TE, ESR, RF, AE \\
\hline Zhang 2017 [13] & yes & unclear & unclear & yes & 4 & GSZT & $\begin{array}{l}\text { LEF } 20 \mathrm{mg} \text { qd, } \\
\text { Nime } 0.1 \mathrm{~g} \text { bid }\end{array}$ & 12 weeks & $\begin{array}{c}\text { TE, DMS, } \\
\text { ESR, RF, CRP }\end{array}$ \\
\hline Yang 2016 [14] & unclear & unclear & unclear & unclear & 2 & GSZT & CXB $0.2 \mathrm{~g} \mathrm{qd}$ & 12 weeks & $\mathrm{TE}$ \\
\hline Tian 2011 [15] & unclear & unclear & unclear & unclear & 2 & GSZT & NSAIDs, GCs & 8 weeks & TE, DMS, ESR \\
\hline Xiao 2012 [16] & unclear & unclear & unclear & unclear & 2 & GSZT & $\begin{array}{l}\text { MTX } 510 \mathrm{mg} \mathrm{qw} \text {, } \\
\text { voltaren } 75 \mathrm{mg} \text { qd }\end{array}$ & 12 weeks & TE, ESR \\
\hline Chen 2017 [17] & yes & unclear & unclear & unclear & 3 & GSZT & $\begin{array}{l}\text { MTX } 10 \mathrm{mg} \mathrm{qw} \text {, } \\
\text { voltaren } 75 \mathrm{mg} \text { qd }\end{array}$ & 12 weeks & $\begin{array}{c}\text { TE, DMS, } \\
\text { ESR, RF, CRP, AE }\end{array}$ \\
\hline Liu 2017 [18] & yes & unclear & unclear & unclear & 3 & GSZT & $\begin{array}{l}\text { LEF } 20 \mathrm{mg} \text { qd, } \\
\text { loxoprofen } 60 \mathrm{mg} \text { tid }\end{array}$ & 8 weeks & ESR, RF, CRP \\
\hline
\end{tabular}

Note: GSZT Guizhi Shaoyao Zhimu Tang, MTX methotrexate, SSZ sulfasalazine, MLX Meloxicam, LEF Leflunomide, Nime nimesulide, $C \mathrm{X} B$ celecoxib, GCS glucocorticoids, $T E$ therapeutic effect, $D M S$ duration of morning stiffness, $E S R$ erythrocyte sedimentation rate, $R F$ rheumatoid factor, $C R \mathrm{P} C$-reactive protein, $A E$ adverse event.

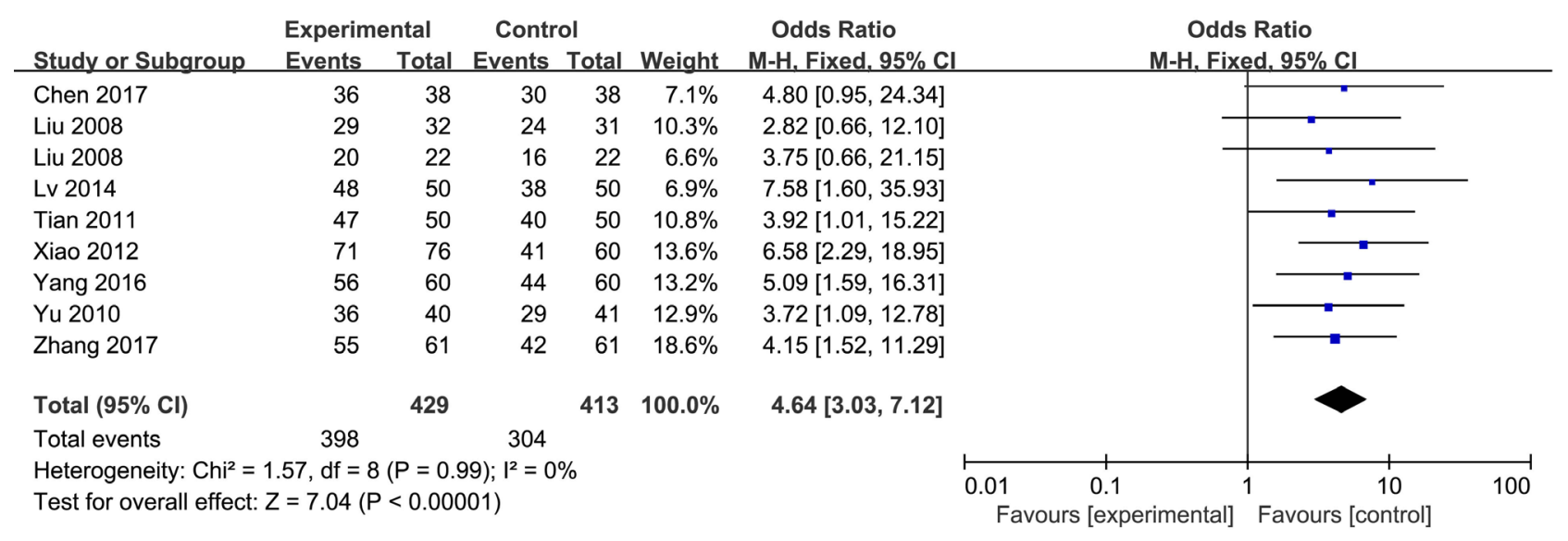

Figure 2. Meta-analysis forest map of the effect comparison.

\subsubsection{Effect on Duration of Morning Stiffness}

Four trials reported the effects on the DMS between the experimental group and control group was carried out. The meta-analysis showed that 4 trials included in the study were homogeneity (no heterogeneity), and showed a insignificant difference $\left(\mathrm{X}^{2}=1.05, \mathrm{P}=0.79, \mathrm{I}^{2}=0 \%\right)$. The fixed effect model was used and showed [MD $=-14.58,95 \% \mathrm{CI}(-16.49,-12.68), \mathrm{Z}=14.99, \mathrm{P}<0.00001$ ] [9] [13] [15] [17]. According to this analysis, it can be considered that there is a statistically significant difference in the effect of the DMS between the combined trial 


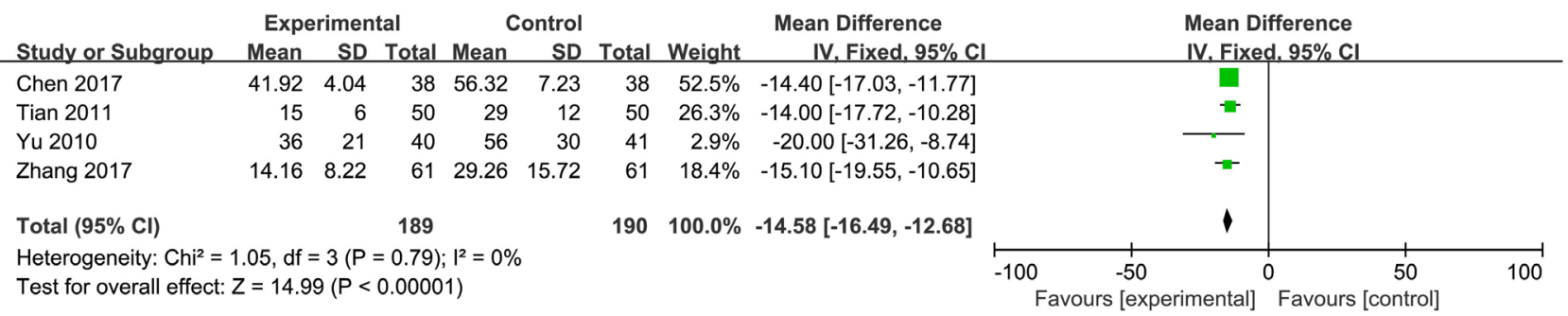

Figure 3. Meta-analysis forest map of the effect on the DMS.

Table 2. Comparison of the improvement of RA on ESR, RF, CRP in the two groups.

\begin{tabular}{|c|c|c|c|c|c|c|c|c|c|c|}
\hline \multirow{2}{*}{$\begin{array}{l}\text { Evaluation } \\
\text { index }\end{array}$} & \multirow{2}{*}{ Trials } & \multicolumn{2}{|c|}{ Interventions } & \multicolumn{3}{|c|}{ Heterogeneity test } & \multirow{2}{*}{$\begin{array}{l}\text { Effect } \\
\text { model }\end{array}$} & \multicolumn{3}{|c|}{ Total effect } \\
\hline & & Experimental & Control & $\mathrm{X}^{2}$ & $\mathrm{I}^{2}$ & $\mathrm{P}$ & & $\mathrm{MD}[95 \% \mathrm{CI}]$ & $\mathrm{Z}$ & $\mathrm{P}$ \\
\hline ESR & 7 & 317 & 318 & 241.67 & $98 \%$ & $<0.001$ & random & $\begin{array}{c}-12.62 \\
{[-18.69,-6.54]}\end{array}$ & 4.07 & $<0.001$ \\
\hline $\mathrm{RF}$ & 4 & 209 & 209 & 219.45 & $99 \%$ & $<0.001$ & random & $\begin{array}{c}-49.88 \\
{[-77.6,-22.09]}\end{array}$ & 3.52 & $<0.001$ \\
\hline CRP & 4 & 199 & 200 & 0.55 & $0 \%$ & 0.91 & fixed & $\begin{array}{c}-5.34 \\
{[-5.86,-4.82]}\end{array}$ & 20.04 & $<0.001$ \\
\hline
\end{tabular}




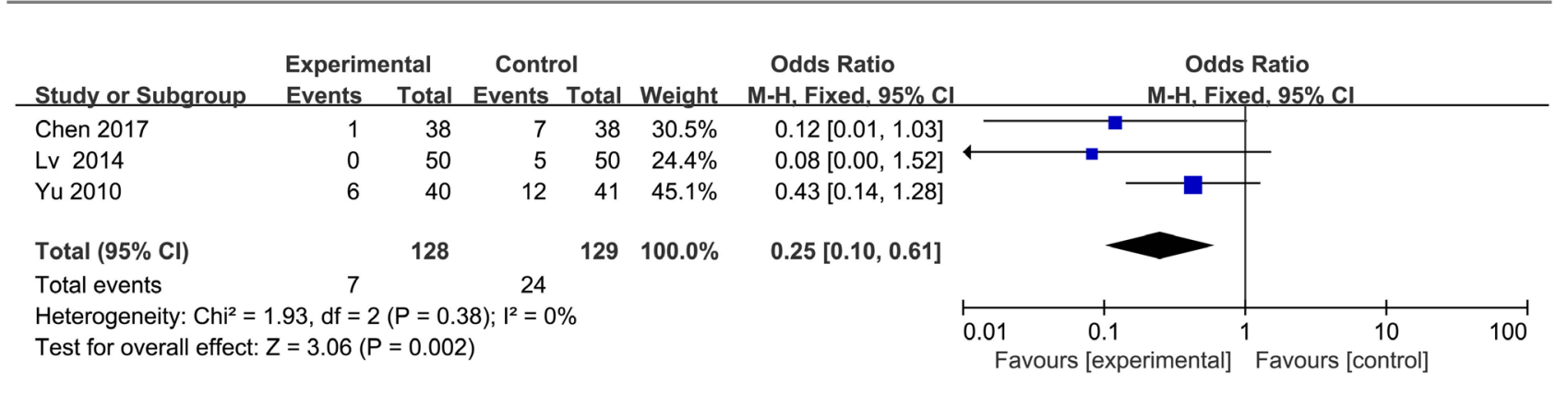

Figure 4. Meta-analysis forest map of the effect on the adverse events.

dizziness, 1 cases of diarrhea and 1 cases of oral ulcer. As shown in Table 3, these side effects, however, which could be alleviated or disappeared after symptomatic treatment in experimental group.

\subsubsection{Publication Bias}

A funnel plot analysis was carried out on the efficacy of the 9 studies in RA, the distribution of each study is symmetrical, and the funnel plot is basically symmetric. It can be concluded that there is no publication bias, and the meta-analysis was credible. See Figure 5.

\section{Discussions}

Guizhi Shaoyao Zhimu Tang, a Chinese medicinal formula, is accepted as the most prevalent and effective treatment for RA in many Asian countries [19]. The essential herbs in this formula, GSZT, are well-known for the book as follow: "Synopsis of Prescriptions of the Golden Chamber". It is the main prescription for the treatment of "arthralgia syndrome". The theory of TCM holds that is "Medicinal and Edible". Modern pharmacological research shows that the formula can significantly reduce the PGE2 content in the inflammatory tissue of adjuvant induced arthritis, inhibit the migration of white blood cells, and reduce the permeability of capillaries [20]. Another study pointed out that the GSZT has similar efficacy to methotrexate in treatment of RA, which could inhibit the secretion of IL-2 and CRP in varying degrees [21]. It also shows the effects in treating RA by adjusting to IL-2, IL-6, IL-17, IL-27, IL-32, TNF- $\alpha$, HRAS, CD14, CD40, and TLR-MAPKs signaling pathway, TLR-NF- $\kappa$ B signaling pathway, NLR signaling pathways and TNF signaling pathway [13] [22]. The animal experiments have also been found that the GSZT can have a protective effect on collagen II-induced arthritis (CIA), and can significantly inhibit the histopathological changes of rheumatoid arthritis and restrain the expression of RANKL/OPG [23] [24]. It is indicated that the GSZT has the function of anti-rheumatism, inhibiting the immune response, and also provides the molecular basis for the treatment of RA. Therefore, the treatment of RA is quite effective in clinic.

This system systematically evaluated the inclusion criteria of clinical studies. The results showed that the GSZT combined with the Western medicine was better than the Western medicine alone in the treatment of RA. According to the funnel plot analysis, this meta-analysis is credible. The experimental group was 
Table 3. Effect of adverse events in the two groups.

\begin{tabular}{cccccccccc}
\hline & \multicolumn{1}{c}{ Adverse events } & & & \\
Interventions & $\begin{array}{c}\text { nausea } \\
\text { vomiting }\end{array}$ & $\begin{array}{c}\text { epigastric } \\
\text { discomfort }\end{array}$ & skin rash & dizziness & $\begin{array}{c}\text { abnormal liver } \\
\text { function }\end{array}$ & leukopenia & diarrhea & $\begin{array}{c}\text { oral ulcer } \\
\text { dry mouth }\end{array}$ & Total \\
\hline Experimenal & 1 & 1 & 1 & 1 & 1 & 0 & 1 & 1 & 7 [9] [17] \\
Control & 6 & 7 & 0 & 0 & 3 & 2 & 0 & 6 & $24[9][12][17]$ \\
\hline
\end{tabular}

Note: 1) The conditions of [9] [12] [17] applied the Western medicine shows in Table 1. 2) The AEs of [9], 1 case of nausea vomiting, 1 case of epigastric discomfort, 1 case of skin rash, 1 case of dizziness, 1 case of abnormal liver function in the experimental group; 4 case of nausea vomiting and epigastric discomfort, 3 case of oral ulcer, 3 case of abnormal liver function, 1 case of leukopenia in the control group. 3) The AEs of [12], no AEs in the experimental groupand 5 case of epigastric discomfort in the control group; 4) The AEs of [17], 1 case of diarrhea in the experimental group; 2 case of nausea vomiting, 2 case of epigastric discomfort, 3 case of dry mouth in the control group.

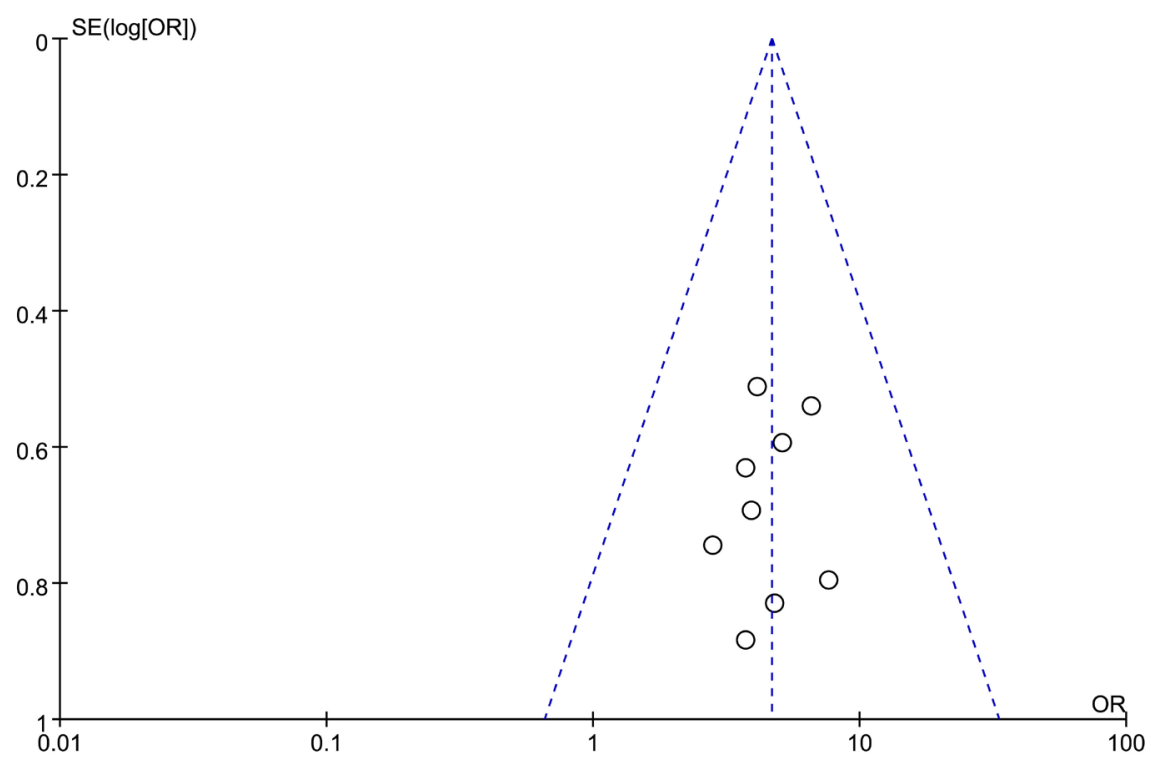

Figure 5. Funnel plot for publication bias in the included trials.

also better than the western medicine treatment group in terms of clinical symptoms, laboratory indicators and adverse reactions. From the large amount of retrieved study, it can be seen that although there are many adverse reactions in the treatment of RA with conventional Western medicine alone, the treatment of western medicine in China is still widely applied due to its exact clinical efficacy and clinical pharmacology. This study confirmed the efficacy and safety that the modified GSZT combined with Western medicine is more effective, regardless of clinical efficacy, or clinical symptoms, laboratory indicators and safety are better, and can better reflect the complementary advantages of Chinese and Western medicine. It would have good clinical promotion and application value.

This study confirmed the efficacy and safety of the GSZT combined with western medicine in the treatment of RA from the perspective of evidence-based medicine. However, through detailed analysis of the articles, the quality of articles was low, and the sample size was also low. Randomization, withdrawal, and loss of follow-up were mostly unknown. They didn't mention the blinded method and allocation concealment, and the specific interventions into the RCT 
dose have different size and duration. The factors certainly influenced the reliability of the result of the meta-analysis. In order to guide clinical practice more scientifically, it is necessary to design a randomized double-blind controlled trial with high quality and provide more reliable evidence-based medical evidence for clinical guidance.

\section{Conflicts of Interest}

The authors declare no conflicts of interest regarding the publication of this paper.

\section{References}

[1] Mcinnes, I.B. and Schett, G. (2011) The Pathogenesis of Rheumatoid Arthritis. NEJM, New England Journal of Medicine, 365, 2205-2219. https://doi.org/10.1056/NEJMra1004965

[2] Smolen, J.S., Aletaha, D. and McInnes, I.B. (2016) Rheumatoid Arthritis. Lancet, 388, 2023-2038. https://doi.org/10.1016/S0140-6736(16)30173-8

[3] Lee, Y.H., Bae, S.C. and Song, G.G. (2011) The Efficacy and Safety of Rituximab for the Treatment of Active Rheumatoid Arthritis: A Systematic Review and Meta-Analysis of Randomized Controlled Trials. Rheumatology International, 31, 1493. https://doi.org/10.1007/s00296-010-1526-y

[4] Peng, Z., Li, J., Yong, H., et al. (2010) Traditional Chinese Medicine in the Treatment of Rheumatoid Arthritis: A General Review. Rheumatology International, 30, 713-718. https://doi.org/10.1007/s00296-010-1370-0

[5] Arnett, F.C., Edworthy, S.M., Bloch, D.A., et al. (1988) The American Rheumatism Association 1987 Revised Criteria for the Classification of Rheumatoid Arthritis. Arthritis \& Rheumatism, 31, 315-324. https://doi.org/10.1002/art.1780310302

[6] Jadad, A.R., Moore, R.A., Carroll, D., et al. (1996) Assessing the Quality of Reports of Randomized Clinical Trials: Is Blinding Necessary? Controlled Clinical Trials, 17, 1-12. https://doi.org/10.1016/0197-2456(95)00134-4

[7] Feng, Z.T., Xu, J., He, G.C., et al. (2017) A Systemic Review and Meta-Analysis of the Clinical Efficacy and Safety of Total Glucosides of Peony Combined with Methotrexate in Rheumatoid Arthritis. Clinical Rheumatology, 37, 35-42. https://doi.org/10.1007/s10067-017-3770-y

[8] Higgins, J.P., Thompson, S.G., Deeks, J.J. and Altman, D.G. (2003) Measuring Inconsistency in Meta-Analyses. BMJ, 327, 557-560. https://doi.org/10.1136/bmj.327.7414.557

[9] Yu, J.H. and Zhang, H. (2010) Traditional Chinese and Western Medicine Treatment of Refractory Rheumatoid Arthritis Clinical Observation. Chinese Journal of Experimental Traditional Medical Formulae, 16, 201-203.

[10] Liu, W.J. (2008) Treatment of 22 Cases of Rheumatoid Arthritis with GSZT. Journal of Practical Traditionail Chinese Internal Medicine, 22, 44.

[11] Liu, M.X. (2008) Treatment of 22 Cases of Active Rheumatoid Arthritis with GSZT. Shandong Journal of Traditional Chinese Medicine, 27, 374-375.

[12] Lv, A.D. (2014) Curative Effect Observation on the Treatment of Rheumatoid Arthritis with GSZT. Modern Chinese and Western Medicine Journal, 23, 393-394.

[13] Zhang, P.K., Wang, F. and Zhang, G.S. (2017) Clinical Observation on Xiao Huo- 
luodan Combined with Guizhi Shaoyao Zhimu Tang for Rheumatic Arthritis with Stagnancy of Cold-Dampness. Chinese Journal of Experimental Traditional Medical Formulae, 23, 181-186.

[14] Yang, A.P. and Wang, X.W. (2016) Treatment of 60 Cases of Rheumatoid Arthritis with GSZT. Hebei Journal of Traditional Chinese Medicine, 38, 562-564.

[15] Tian, M.L. (2011) Treatment of 100 Cases of Rheumatoid Arthritis with GSZT. Journal of China Traditional Chinese Medicine Information, 3, 153-227.

[16] Xiao, S.H. (2012) Clinical Observation of Guizhi Shaoyao Zhimu Decoction in Treatment of 36 Cases with Active Rheumatoid Arthritis. China Modern Medicine, 19, 112-115.

[17] Chen, Z.L. and Zhang, S. (2017) Curative Effect Observation on the Treatment of Rheumatoid Arthritis Treated with GSZT. Chinese Traditional Patent Medicine, 39, 1539-1541.

[18] Liu, X.W. (2017) Effect of Routine Western Medicine and Guizhi Shaoyao Zhimu Decoction on Patients with Rheumatoid Arthritis. Chinese Archives of Traditional Chinese Medicine, 35, 504-507.

[19] Setty, A.R. and Sigal, L.H. (2005) Herbal Medications Commonly Used in the Practice of Rheumatology: Mechanisms of Action, Efficacy, and Side Effects. Seminars in Arthritis and Rheumatism, 34, 773-784. https://doi.org/10.1016/j.semarthrit.2005.01.011

[20] Xv, J.L., Luo, X.S. and Zhang, C.G. (2003) The Study on the Pharmacodynamics of GSZT in Antirheumatism. Journal of Chinese Medicinal Materials, 26, 662-664.

[21] Zhang, Q., Wu, H., Jiang, Y., et al. (2006) The Effect of GSZT on the Proliferation of Collagen Induced Arthritis T Lymphocyte in Transgenic Mice. Journal of Chengdu University of TCM, 29, 24-26.

[22] Zheng, M.S., Jiang, Q.Y., et al. (2017) Mechanism of Guizhi Shaoyao Zhimu Tang in Treatment of Rheumatoid Arthritis Based on Bioinformatics. Chinese Journal of Experimental Traditional Medical Formulae, No. 10, 195-200.

[23] Chen, H., He, X.J. and Xv, J.H. (2012) Effect of GSZT on Pathological Changes of Ankle Joints in Rats with Collagen II-Induced Arthritis. China Journal of Basic Medicine in Traditional Chinese Medicine, No. 18, 555-559.

[24] Yu, J.L. and Zhang, J. (2013) Effect of Guishao Zhimu Decoction on Histiocyte RANKL/OPG of Collagen-Induced Arthritis. Journal of Liaoning University of TCM, No. 6, 24-25. 